\title{
Investigating State Restriction in Fluorescent Protein FRET Using Time-Resolved Fluorescence and Anisotropy
}

Thomas S. Blacker, ${ }^{\dagger,}, \S$ WeiYue Chen, ${ }^{\| \odot}$ Edward Avezov, ${ }^{\perp}$ Richard J. Marsh, ${ }^{\dagger, \#}$ Michael R. Duchen, ${ }^{\S}$ Clemens F. Kaminski, " and Angus J. Bain ${ }^{*, \dagger, \ddagger \odot}$

${ }^{\dagger}$ Department of Physics \& Astronomy, ${ }^{\ddagger}$ Centre for Mathematics and Physics in the Life Sciences and Experimental Biology, and ${ }^{\S}$ Department of Cell \& Developmental Biology, University College London, Gower Street, London WC1E 6BT, United Kingdom

"Department of Chemical Engineering and Biotechnology, University of Cambridge, Pembroke Street, Cambridge CB2 3RA, United Kingdom

${ }^{\perp}$ Cambridge Institute for Medical Research, University of Cambridge, Cambridge CB2 0XY, United Kingdom

Supporting Information

ABSTRACT: Most fluorescent proteins exhibit multiexponential fluorescence decays, indicating a heterogeneous excited state population. FRET between fluorescent proteins should therefore involve multiple energy transfer pathways. We recently demonstrated the FRET pathways between EGFP and mCherry $(\mathrm{mC})$, upon the dimerization of 3-phosphoinositide dependent protein kinase 1 (PDK1), to be highly restricted. A mechanism for FRET restriction based on a highly unfavorable $\kappa^{2}$ orientation factor arising from differences in donor-acceptor transition dipole moment angles in a far from coplanar and near static interaction geometry was proposed. Here this is tested via FRET to $\mathrm{mC}$ arising from the association of glutathione (GSH) and glutathione S-transferase

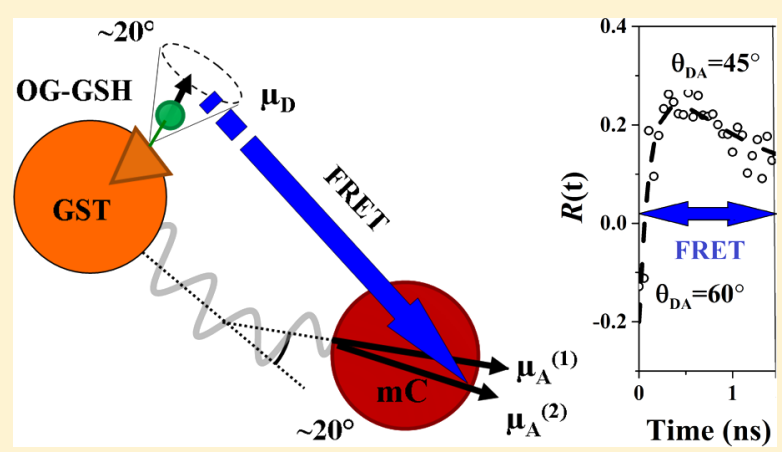
(GST) with an intrinsically homogeneous and more mobile donor Oregon Green 488 (OG). A new analysis of the acceptor window intensity, based on the turnover point of the sensitized fluorescence, is combined with donor window intensity and anisotropy measurements which show that unrestricted FRET to $\mathrm{mC}$ takes place. However, a long-lived anisotropy decay component in the donor window reveals a GST-GSH population in which FRET does not occur, explaining previous discrepancies between quantitative FRET measurements of GST-GSH association and their accepted values. This reinforces the importance of the local donor-acceptor environment in mediating energy transfer and the need to perform spectrally resolved intensity and anisotropy decay measurements in the accurate quantification of fluorescent protein FRET.

\section{INTRODUCTION}

Förster Resonance Energy Transfer (FRET) describes the nonradiative transmission of electronic energy from a donor molecule to a nearby acceptor due to dipole-dipole coupling. ${ }^{1,2}$ FRET measurements have found widespread application in the study of nanoscale processes in the biosciences, such as changes in conformation and intermolecular interactions. ${ }^{3,4}$ FRET is well understood for homogeneous populations of donors and acceptors, ${ }^{2,5}$ but in recent years, the use of genetically encodable fluorescent protein FRET pairs has become widespread. ${ }^{6}$ Many fluorescent proteins exhibit multiexponential fluorescence decay kinetics, $^{7-9}$ indicating the existence of multiple emitting states, molecular conformations, or local environments. Noninteracting populations in fluorescent protein FRET have previously been recognized. ${ }^{10-12}$ However, it remains an open question as to whether these arise as an intrinsic property of the dipoledipole interaction, environmental heterogeneity such as variations in the FRET interaction geometry, or misfolding leading to the production of subpopulations of chromophores incapable of participating in FRET. ${ }^{10,13}$

Accurate quantitative application of fluorescent protein FRET is crucially dependent on the correct understanding of the underlying photophysics. This point is strongly evidenced by our recent work on the homodimerization of 3phosphoinositide dependent kinase-1 (PDK1) using the standard FRET pair of enhanced green fluorescent protein (EGFP) and mCherry $(\mathrm{mC}) .{ }^{14}$ Both proteins exhibit intrinsic biexponential fluorescence decays. ${ }^{15}$ Combining time-resolved fluorescence intensity and anisotropy measurements of the donor and acceptor, we found that FRET was highly restricted, involving transfer from only one emitting state of EGFP to the minority decay component of $\mathrm{mC}$. In contrast, when emulating unrestricted FRET by the optical excitation of $\mathrm{mC}$ across the donor-acceptor spectral overlap, no such constraint was

Received: November 8, 2016

Revised: December 23, 2016

Published: December 29, 2016 
observed. ${ }^{15}$ It was calculated that conventional intensity based FRET techniques, ${ }^{16-21}$ which would not report this restriction, would lead to an underestimation of the true PDK1 interacting fraction by over an order of magnitude. ${ }^{14}$

We proposed two mechanisms for the FRET restrictions between EGFP and $\mathrm{mC}^{15}$ First, that the intrinsic energy transfer rates for the two donor (EGFP) populations were widely dissimilar. Given close fluorescence lifetimes for the two populations (2.4 ns and $\left.3.1 \mathrm{~ns}^{15}\right)$ this hypothesis would imply a low radiative rate coupled with a "compensating" fast nonradiative decay channel for the FRET inactive donors. However, precision measurements of the stimulated emission depletion (STED) dynamics in recombinant EGFP in our group indicate that both emitting populations have strong transition dipole moments. ${ }^{22}$ This mitigates against a significant difference in the radiative decay rates of the two populations. The second mechanism recognized that, in a far from coplanar FRET interaction geometry, small differences in the relative donor-acceptor transition dipole moment angles for the two populations would give rise to a large disparity in the $\kappa^{2}$ orientation parameters ${ }^{23}$ and the corresponding FRET rates.

Here we test the second hypothesis by probing FRET to $\mathrm{mC}$ in a system where EGFP is replaced by the synthetic fluorophore Oregon Green $488^{24}$ (OG). OG is spectrally similar to EGFP but is characterized by a monoexponential fluorescence lifetime of $\sim 4 \mathrm{~ns}^{25}$ Moreover, given its considerably smaller hydrodynamic volume and molecular weight compared to EGFP ( $880 \AA^{3}$ and $0.5 \mathrm{kDa}$ vs $58000 \AA^{3}$ and $29 \mathrm{kDa}^{25-27}$ ), OG displays a significantly higher degree of orientational mobility. FRET with OG as opposed to EGFP should therefore, in principle, be characterized by less complex population dynamics and should sample a greater range of donor-acceptor orientations.

Fluorescence Dynamics in FRET between Oregon Green and mCherry. The FRET system studied here consists of $\mathrm{mC}$ fused to the enzyme glutathione S-transferase (GST) and its substrate, glutathione (GSH), attached to OG. FRET between $\mathrm{OG}$ and $\mathrm{mC}$ can occur when GSH attaches to its binding site on GST. The affinity of GSH for GST is sufficiently high $\left(K_{\mathrm{D}} \approx 20 \mu \mathrm{M}^{28}\right)$ that their binding is routinely exploited in the purification of recombinant proteins, ${ }^{29,30}$ ensuring a significant population of donor-acceptor complexes for FRET measurements. An overview of the fluorescence and FRET dynamics in the OG-GSH/GST-mC system is illustrated in Figure 1. Fluorescence following two-photon excitation of OG at $880 \mathrm{~nm}$ is detected in two spectral windows: $515-540$ $\mathrm{nm}$ (the donor window $\Delta \lambda_{\mathrm{D}}$ ) and 630-650 $\mathrm{nm}$ (the acceptor window $\Delta \lambda_{\mathrm{A}}$ ). The contribution of $\mathrm{mC}$ fluorescence in the donor window is negligible (see Supporting Information Appendix S1); however, there is unavoidable "bleed through" of OG emission superimposed on the sensitized $\mathrm{mC}$ emission arising from FRET (see Figure 1). Characterization of the intrinsic fluorescence and anisotropy properties of OG-GSH (donor) and GST-mC (acceptor) is thus an essential first step in the analysis of the energy transfer dynamics of these molecules.

\section{MATERIALS AND METHODS}

Fluorescent Probes. Production of OG-GSH and recombinant GST-mC has been detailed elsewhere. ${ }^{24}$ In this work, FRET was studied in mixtures of approximately $60 \mu \mathrm{M}$ OG-GSH and $30 \mu \mathrm{M}$ GST-mC in phosphate buffered saline at $\mathrm{pH}$ 7.4. Given a $K_{\mathrm{D}}$ of $20 \mu \mathrm{M}$ for the GSH-GST interaction,
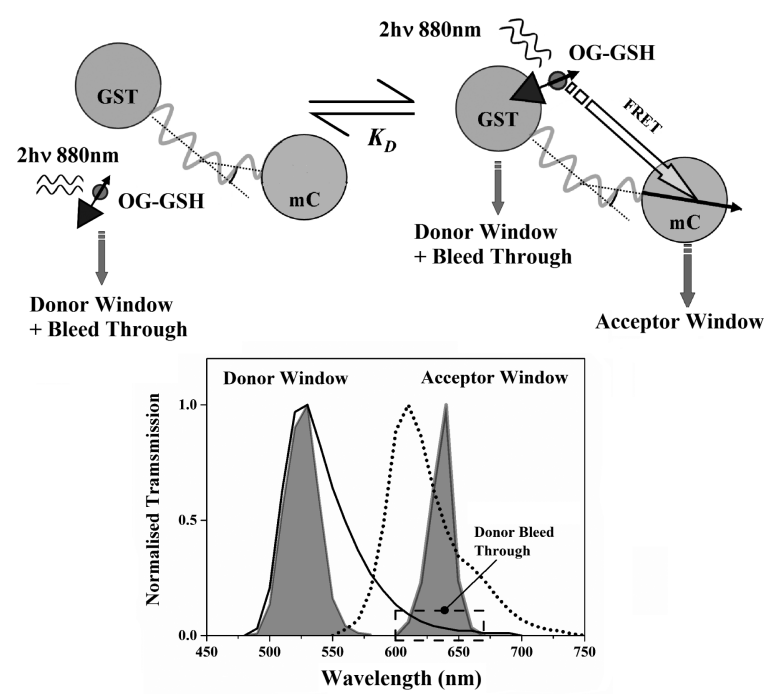

Figure 1. Illustration of the fluorescence dynamics between OG and $\mathrm{mC}$ arising from the association of GSH and GST. Free and bound OG-GSH is excited by two-photon excitation at $880 \mathrm{~nm}$. This causes intrinsic OG and sensitized (FRET excited) $\mathrm{mC}$ fluorescence which collectively span $490-750 \mathrm{~nm}$. Time-resolved fluorescence intensity and anisotropy measurements are made in two spectral windows illustrated by the gray areas (filter transmission curves) superimposed on the OG (solid line) and $\mathrm{mC}$ (dotted line) emission spectra. Donor window measurements report solely OG fluorescence dynamics (spontaneous emission from free OG-GSH and spontaneous emission plus nonradiative FRET transfer from OG-GSH-GST-mC). The contribution from OG (donor) bleed through in the acceptor window leads to more complex intensity and anisotropy dynamics, necessitating the new approaches applied in this work.

this implied that approximately a quarter of the total OG-GSH would be part of a complex with GST-mC. ${ }^{28}$ Photophysical characterization of the isolated donor and acceptor molecules was performed on solutions of approximately $10 \mu \mathrm{M}$ OG-GSH and $4 \mu \mathrm{M}$ GST-mC, respectively. For two-photon characterization (see Supporting Information Appendix S2), unconjugated OG was obtained from Life Technologies (Paisley, UK).

Fluorescence Intensity and Anisotropy Measurements. Time-resolved fluorescence measurements were performed using time-correlated single photon counting $(\mathrm{TCSPC})^{31}$ using an apparatus described previously. ${ }^{15,32}$ Experimental procedures are detailed in Supporting Information Appendix S3. Fluorescence intensity and anisotropy analysis followed established protocols ${ }^{15,32}$ (see Supporting Information Appendix S4).

\section{RESULTS AND DISCUSSION}

OG-GST and GST-mC: Intrinsic Fluorescence and Anisotropy Dynamics. Measurements of the fundamental population and rotational dynamics of the isolated donor and acceptor molecules are detailed in Supporting Information Appendix S5. Akin to OG in solution, ${ }^{25}$ OG-GSH fluorescence decayed with a single lifetime of $4.26( \pm 0.06)$ ns and yielded rotational correlation times of $0.251( \pm 0.008) \mathrm{ns}$ and $0.279( \pm 0.003)$ ns with single-photon and two-photon excitation, respectively. Measurements emulating unrestricted FRET to GST-mC used single photon excitation at wavelengths spanning the donor-acceptor spectral overlap with fluorescence detection in the acceptor window (Figure 1). 
Across this range, GST-mC exhibited a biexponential fluorescence decay with a constant mean lifetime of $1.564( \pm 0.002) \mathrm{ns}$, in line with previous measurements of recombinant $\mathrm{mC} .^{15}$ Attachment to GST leads to a less marked disparity in the short and long lifetimes, referred hereafter as states 1 and 2, respectively, with overlap-weighted average values of $1.315( \pm 0.002)$ ns and $1.897( \pm 0.003)$ ns, with $57.2( \pm 0.2) \%$ of GST-mC in the short lifetime state, lower than for $\mathrm{mC}$ alone. ${ }^{15}$ Weighted by the spectral overlap, the fluorescence anisotropy of GST-mC decayed with a minority amplitude fast decay component of $1.1( \pm 0.1) \mathrm{ns}$ and a majority component with a longer rotational correlation time of $28( \pm 1)$ ns. Rigid body rotational diffusion, where each of these components corresponds to the motion of a distinct species, was ruled out by poor fits of this composite anisotropy model ${ }^{32}$ (Supporting Information Equation S6, $\chi_{\mathrm{R}}{ }^{2}=4.8 \pm 0.5$ ) to the data. Models of restricted rotational diffusion of $\mathrm{mC}$ relative to GST were found to be more appropriate, with the fitting parameters indicating constrained $\mathrm{mC}$ motion within an angular range of between $15^{\circ}$ and $20^{\circ}$ (see Supporting Information Appendix S5).

Donor Window Intensity and Anisotropy Decays in the Presence of FRET. The differential equations describing OG-mC FRET and their solutions are similar in form to our previous work ${ }^{15}$ and are set out in Supporting Information Appendix S6. Following two-photon excitation of the OGGSH/GST-mC mixture at $880 \mathrm{~nm}$, at which direct acceptor excitation can be neglected (Supporting Information Appendix S2), detection of fluorescence in the donor window should in principle yield decay dynamics described by

$$
\begin{aligned}
I\left(t, \Delta \lambda_{\mathrm{D}}\right) \propto & F_{1}^{\mathrm{I}} \exp \left(-\left(k_{\mathrm{F}}^{\mathrm{D}}+k_{\mathrm{FRET} 1}\right) t\right) \\
& +F_{2}^{\mathrm{I}} \exp \left(-\left(k_{\mathrm{F}}^{\mathrm{D}}+k_{\mathrm{FRET} 2}\right) t\right)+\left(1-F_{1}^{\mathrm{I}}-F_{2}^{\mathrm{I}}\right) \exp \left(-k_{\mathrm{F}}^{\mathrm{D}} t\right)
\end{aligned}
$$

Here $F_{1}{ }^{\mathrm{I}}$ and $F_{2}{ }^{\mathrm{I}}$ are the fraction of donors participating in FRET to the two emitting states of $\mathrm{mC}$ with corresponding FRET rates $k_{\text {FRET1 }}$ and $k_{\text {FRET2 }}$. Single-, two-, and threecomponent fits to the data (Figure 2) were performed. From the weighted residuals it was clear that a single exponential decay was a poor description of the fluorescence dynamics. The three-component fit gave a slightly lower $\chi_{\mathrm{R}}^{2}$ value than that for the two-component model (1.4 vs 1.8). However, the uncertainties in the fitted values were unacceptably large, suggesting overparametrization. ${ }^{33}$ The two-component fit extracted lifetimes of $4.213( \pm 0.009)$ ns and 1.33( \pm 0.07$)$ ns. By comparison with the fluorescence lifetime measurements on pure OG-GSH, it was clear the former corresponded to noninteracting $\mathrm{OG}-\mathrm{GSH}, \tau_{\mathrm{DNI}}=1 / k_{\mathrm{F}}{ }^{\mathrm{D}}$ with the latter corresponding to the OG-GSH population undergoing FRET. This suggested a total interacting fraction $F_{1}{ }^{\mathrm{I}}+\mathrm{F}_{2}{ }^{\mathrm{I}}=$ $0.136( \pm 0.005)$, based on the relative amplitudes of the decay components. From eq 1, two interacting donor lifetimes are expected, arising from FRET to each of the two states in GST$\mathrm{mC}$, corresponding to $\tau_{\mathrm{D} 1}=1 /\left(k_{\mathrm{F}}{ }^{\mathrm{D}}+k_{\mathrm{FRET} 1}\right)$ and $\tau_{\mathrm{D} 2}=1 /$ $\left(k_{\mathrm{F}}^{\mathrm{D}}+k_{\mathrm{FRET} 2}\right)$. The recovery of only one interacting lifetime corresponds to one of two possible scenarios. First, that FRET to $\mathrm{mC}$ had taken place exclusively to one state, either the short lifetime state $\left(k_{\mathrm{FRET} 2}=0\right.$ and ${F_{2}}^{\mathrm{I}}=0)$ or the longer lived state $\left(k_{\text {FRET1 }}=0\right.$ and $\left.F_{1}{ }^{1}=0\right)$ or, second, that the rates of energy transfer to both states were sufficiently close as to be indistinguishable in the overall fluorescence decay.

The donor window fluorescence anisotropy should, in principle, contain population weighted contributions from
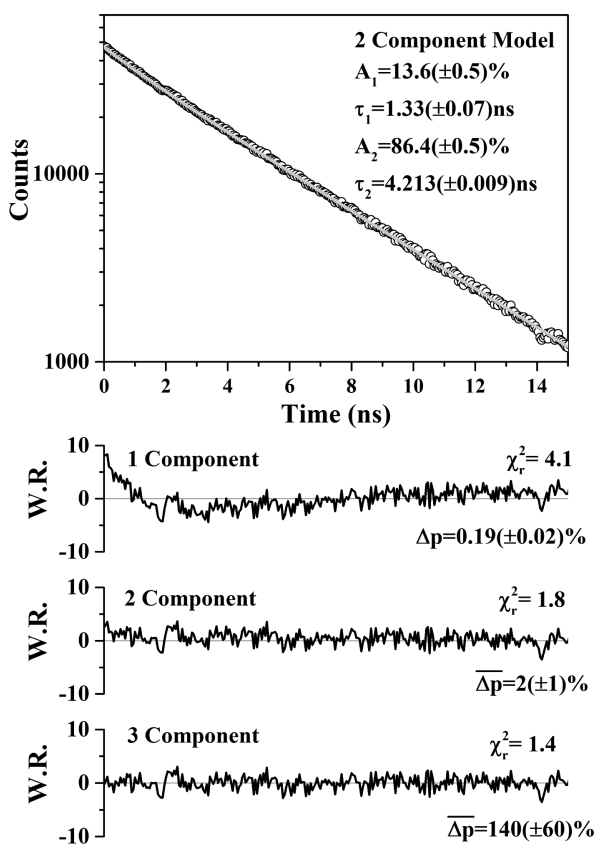

Figure 2. Donor window fluorescence intensity decay of a mixture of $60 \mu \mathrm{M}$ OG-GSH and $30 \mu \mathrm{M}$ GST-mC with $880 \mathrm{~nm}$ excitation. The decay departs from the single exponential found for OG-GSH and is best fit to a biexponential decay with the $1.33 \mathrm{~ns}$ lifetime corresponding to the OG population undergoing FRET with an interacting fraction $F_{\mathrm{I}}=\left(F_{1}{ }^{\mathrm{I}}+F_{2}{ }^{\mathrm{I}}\right)=0.136( \pm 0.005)$. The lifetimes yield a FRET rate of $0.51( \pm 0.04) \mathrm{ns}^{-1}$.

noninteracting freely rotating OG-GSH and a fast lifetime but more slowly rotating component arising from energy transfer within the OG-GSH-GST-mC complex. Due to the fast transfer dynamics $\left(k_{\mathrm{FRET}}=0.5 \mathrm{~ns}^{-1}\right)$, the contribution of the latter to the total fluorescence signal by 2 ns after excitation was calculated to be less than 6\% (see Supporting Information Appendix S7). Emission in the donor window should therefore be dominated by noninteracting OG. The measured anisotropy decay is shown in Figure 3, where it can be seen in the inset that FRET does not alter the early time dynamics as would be expected for rapid large angle acceptor motion. ${ }^{34,35}$ The longer lived anisotropy decay component observed in the OG-GSH GST-mC solution implies the presence of a subpopulation of associating but FRET inactive GSH and GST. An inhomogeneous OG-GSH population is indicated by the poor fit of homogeneous single and double exponential decays to $R(t)$ seen from the weighted residuals. The fluorescence anisotropy of a heterogeneous (composite) system is given by a time dependent weighted sum of the individual anisotropies (Supporting Information Equation S6) ${ }^{15,36}$

$$
\begin{aligned}
R(t)= & R_{\mathrm{OG}}^{\text {bound }}(t)\left[F_{\mathrm{I}} W_{\mathrm{I}}(t)+\left(1-F_{\mathrm{I}}\right)\left(1-\eta_{\text {free }}\right) W_{\mathrm{NI}}(t)\right] \\
& +R_{\mathrm{OG}}^{\text {free }}(t)\left(1-F_{\mathrm{I}}\right) \eta_{\text {free }} W_{\mathrm{NI}}(t)
\end{aligned}
$$

where the $W_{\mathrm{I}}(t)$ and $W_{\mathrm{NI}}(t)$ are the time dependent weighting factors of the interacting and noninteracting OG-GSH populations, respectively (Supporting Information Equation S7), and $\eta_{\text {free }}$ denotes the fraction of noninteracting OG-GSH that is not bound to GST-mC. The anisotropy is best fit by this model when $R_{\mathrm{OG}}{ }^{\text {bound }}(t)$ corresponds to the restricted rotational diffusion of OG with a cone angle of $25( \pm 6)^{\circ}$, a local diffusion coefficient $D=0.10( \pm 0.05) \mathrm{ns}^{-1}$, and $\eta_{\text {free }}=$ 

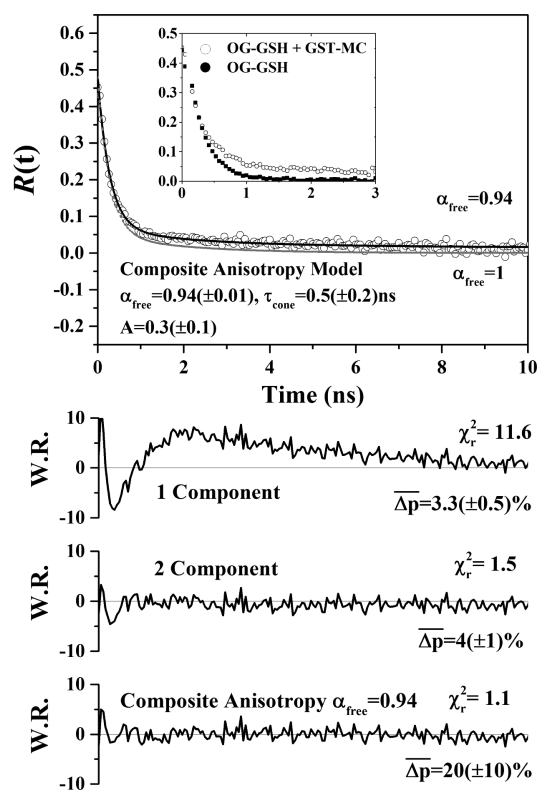

Figure 3. Donor window fluorescence anisotropy of OG-GSH. The inset is a comparison of the early time anisotropy for a pure solution of OG-GSH and the OG-GSH GST-mC mixture. $R(t)$ is best described by a composite anisotropy model (black line) in which $6( \pm 1) \%$ of the noninteracting OG-GSH is bound to GST-mC. A fully nonbound noninteracting fraction $\left(\eta_{\text {free }}=1\right)$ is unable to reproduce the observed anisotropy (gray line).

$0.94( \pm 0.01)$. A full description of the analysis is set out in the Supporting Information Appendix S7.

The environment of OG-GSH and its local motion when attached to GST is less constrained and more rapid than for EGFP in the PDK1 FRET system $\left(25^{\circ}\right.$ vs $15^{\circ}$ and $0.1 \mathrm{~ns}^{-1}$ vs $\left.0.01 \mathrm{~ns}^{-1}\right) .{ }^{15}$ Given an interacting fraction of $13.6( \pm 0.5) \%$ (Figure 2$), 86.4( \pm 0.5) \%$ of the total OG-GSH population is FRET inactive. From the composite anisotropy analysis, $6( \pm 1) \%$ of this population corresponded to FRET inactive OG-GSH bound to GSH. This is a significant result, as while this corresponds to just $5.2( \pm 0.9) \%$ of the total OG-GSH population, it represents $28( \pm 3) \%$ of the total bound OG-GSH population. Thus, despite the increase in conformational freedom, FRET does not occur in these complexes. It should be noted that conventional intensity decay measurements are insensitive to this phenomenon, which contribute to overestimates of the dissociation constant of GSH and GST previously made using this FRET pair ${ }^{24}$ (see Supporting Information Appendix S8).

Noninteracting fluorescent protein populations have been observed in previous studies and have been ascribed to the presence of unmatured $\mathrm{mC}$ or photoconversion of its chromophore. $^{10,37,38}$ From our recent work, we know that restricted FRET to only one of the two emitting $\mathrm{mC}$ populations is possible. ${ }^{15}$ To fully investigate the nature of the incomplete FRET between $\mathrm{OG}$ and $\mathrm{mC}$, fluorescence intensity and anisotropy measurements in the acceptor window are crucial.

Acceptor Window Intensity and Anisotropy Measurements. As depicted in Figure 1, the composite fluorescence in the acceptor window arises from both sensitized acceptor fluorescence and the bleed through from interacting and noninteracting donor populations. ${ }^{15}$ The population dynamics that contribute to the acceptor window fluorescence are set out in detail in Supporting Information Appendix S6. With the possibility of FRET to two states in $\mathrm{mC}$, the acceptor window intensity decay $I\left(t, \Delta \lambda_{A}\right)$ has the form

$$
\begin{aligned}
I\left(t, \Delta \lambda_{\mathrm{A}}\right) \propto & F_{1}^{\mathrm{I}} \exp \left(-\left(k_{\mathrm{F}}^{\mathrm{D}}+k_{\mathrm{FRET} 1}\right) t\right)+F_{2}^{\mathrm{I}} \exp \left(-\left(k_{\mathrm{F}}^{\mathrm{D}}+k_{\mathrm{FRET} 2}\right) t\right) \\
& +B\left(\Delta \lambda_{\mathrm{A}}\right) F_{1}^{\mathrm{I}} X_{1}\left[\exp \left(-k_{\mathrm{F}}^{\mathrm{A} 1} t\right)-\exp \left(-\left(k_{\mathrm{F}}^{\mathrm{D}}+k_{\mathrm{FRET} 1}\right) t\right)\right] \\
& +B\left(\Delta \lambda_{\mathrm{A}}\right) F_{2}^{\mathrm{I}} X_{2}\left[\exp \left(-k_{\mathrm{F}}^{\mathrm{A} 2} t\right)-\exp \left(-\left(k_{\mathrm{F}}^{\mathrm{D}}+k_{\mathrm{FRET} 2}\right) t\right)\right] \\
& +\left(1-F_{1}^{\mathrm{I}}-F_{2}^{\mathrm{I}}\right) \exp \left(-k_{\mathrm{F}}^{\mathrm{D}} t\right)
\end{aligned}
$$

$B\left(\Delta \lambda_{\mathrm{A}}\right)$ quantifies the proportion of acceptor fluorescence detected relative to the donor, ${ }^{15}$ and $X_{i}$ is the FRET amplitude to state $i$ with fluorescence decay rate $k_{\mathrm{F}}{ }^{\mathrm{A} i}$.

$$
X_{i}=\frac{k_{\mathrm{FRETi}}}{k_{\mathrm{F}}^{\mathrm{D}}+k_{\mathrm{FRETi}}-k_{\mathrm{F}}^{\mathrm{A} i}}
$$

For states with fluorescence lifetimes that vary over the donoracceptor spectral overlap, $X_{1}$ and $X_{2}$ can be modeled as possessing a single lifetime calculated from the overlapweighted averages ${ }^{15}$ (see Supporting Information Appendix S9). The first two terms in eq 3 correspond to the bleed through of interacting donor fluorescence, the third and fourth describe the growth and decay the acceptor emission as a result of FRET transfer, and the final term corresponds to the bleed through of the noninteracting donor fluorescence. In practice the interacting donor bleed through terms have a lower weighting relative to the noninteracting donor bleed through $\left(F_{1}{ }^{\mathrm{I}}+F_{2}{ }^{\mathrm{I}}=0.136\right.$ vs $\left.1-F_{1}{ }^{\mathrm{I}}-F_{2}{ }^{\mathrm{I}}=0.864\right)$ and only contribute to the fluorescence at early times. However, even with prior knowledge of some parameters (e.g., $k_{\mathrm{F}}{ }^{\mathrm{D}}, F_{1}{ }^{\mathrm{I}}+F_{2}{ }^{\mathrm{I}}, k_{\mathrm{F}}{ }^{\mathrm{A} 1}, k_{\mathrm{F}}{ }^{\mathrm{A} 2}$ ), the number of independent decay components involved cannot be resolved reliably, even with good quality time-resolved fluorescence data. ${ }^{33}$ To obtain a fuller picture of the FRET dynamics, the measurement of additional fluorescence observables is necessary. ${ }^{15}$ In our study of FRET between EGFP and $\mathrm{mC}$ arising from PDK1 homodimerization this was provided by the acceptor window fluorescence anisotropy. ${ }^{15}$ The large value of the donor-acceptor angle $\theta_{\mathrm{DA}}\left(65^{\circ}\right)$ and the slow rotational diffusion times of both interacting and noninteracting species relative to the energy transfer and excited state lifetimes $\left(k_{\text {rot }} \approx 0.05 \mathrm{~ns}^{-1}\right.$ vs $k_{\text {FRET }} \approx 0.2 \mathrm{~ns}^{-1}$ and $k_{\mathrm{F}} \approx 0.4 \mathrm{~ns}^{-1}$ ) gave rise to an acceptor window anisotropy that principally depended on the time dependent weighting of a positive anisotropy from the noninteracting donor population with that of the negative anisotropy created by FRET to the acceptor. ${ }^{15} \mathrm{Had}$ FRET to $\mathrm{mC}$ been unrestricted, the anisotropy, while exhibiting a rapid drop due to FRET (observed) as the intensity weightings of EGFP and $\mathrm{mC}$ became comparable, would have been followed by a subsequent rise as the donor weighting became dominant due to the significantly longer average lifetime of EGFP compared to $\mathrm{mC}$ (2.75 ns vs 1.57 ns). ${ }^{15}$ The absence of this rise in the anisotropy could only occur if FRET had exclusively populated the minority (longer lived) $\mathrm{mC}$ species. With OG-GSH/GST-mC, however, the rotational depolarization of the noninteracting donor emission, FRET, and the local motion of OG in the GSH-GST complex all occur on comparable time scales. Consequently the acceptor window anisotropy could not on its own be used as an unequivocal measure of the population dynamics of FRET transfer.

Despite the underlying complexity of the acceptor window intensity signal (eq 3), we have found that a biexponential rise (negative amplitude component) and decay (positive amplitude 
component) model provides the most accurate description of the measured data possible, even with high signal-to-noise levels. ${ }^{15,39}$ In the current system, as shown in Figure 4, we
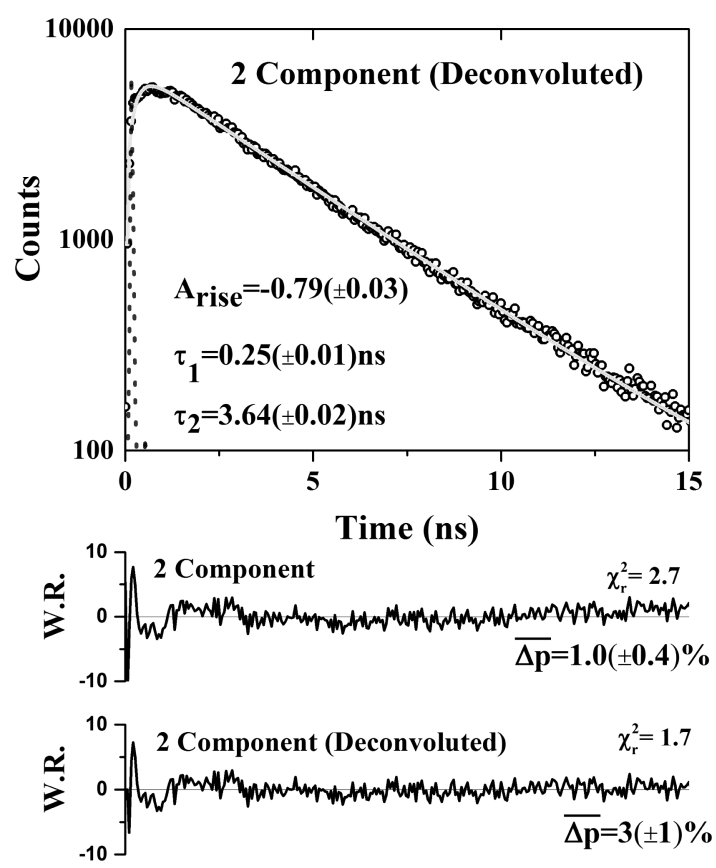

Figure 4. Fluorescence intensity decay of a mixture of $60 \mu \mathrm{M}$ OGGSH and $30 \mu \mathrm{M}$ GST-mC with $880 \mathrm{~nm}$ excitation and detection in the acceptor window. Biexponential fitting yields rise and decay times of $0.25( \pm 0.01)$ ns and $3.64( \pm 0.02)$ ns, respectively. The fit parameters imply a turning point of the data set of $0.66( \pm 0.02) \mathrm{ns}$.

observe a rise lifetime of $\tau_{\text {rise }}=0.25( \pm 0.01) \mathrm{ns}$, a decay lifetime of $\tau_{\text {decay }}=3.64( \pm 0.02) \mathrm{ns}$, and ratio of rise to decay amplitudes of $\left|A_{\text {rise }}\right|=0.79( \pm 0.03)$. Despite this fit accurately describing the shape of the decay, with $\chi_{\mathrm{R}}^{2}=1.7$ and mean parameter uncertainties of $3( \pm 1) \%$, previous work in our group has shown that it is not possible to relate these quantities to the underlying parameters describing the FRET interaction, with the degree of noninteracting bleed through (here around $70 \%$ of the measured signal, see Supporting Information Appendix S10) playing a significant role in distorting the rise and decay times in the biexponential fits and their corresponding amplitudes (see Supporting Information Appendix S11). ${ }^{39}$ However, the time at which the sensitized fluorescence plus donor bleed through reached its maximum value, or turnover point, could be directly related to the FRET parameters. This physically significant feature corresponds to the point at which the rate of increase in the fluorescence intensity due to FRET equals the rate of decrease of fluorescence due to emission by both sensitized acceptors and donor bleed through. This position is therefore a function of all the parameters describing the FRET interaction, allowing the determination of unknown parameter values from the output of a simple biexponential fit. ${ }^{39}$

Plots of eq 3 for FRET to only state 1 or state 2 , or in equal proportion to both states, are shown in Figure 5A. The turning points of the curves were seen to be sensitive to the proportion of FRET to each state. FRET to only the short lifetime $\mathrm{mC}$ state resulted in a turnover point at $0.58 \mathrm{~ns}$, whereas FRET to only the long lifetime state caused a turnover at $0.72 \mathrm{~ns}$. Equal FRET to both states resulted in a turnover point halfway between the two. As such, it appeared that there was a linear

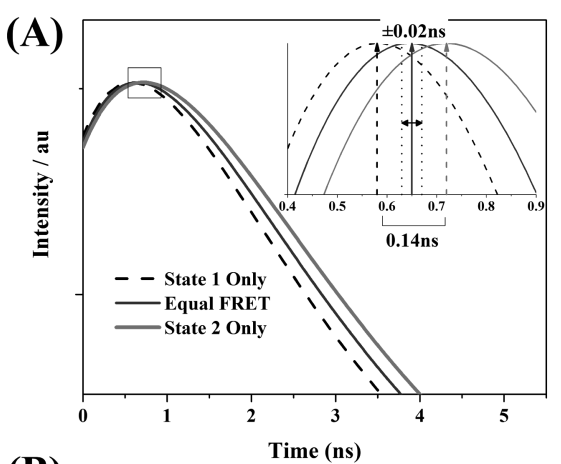

(B)

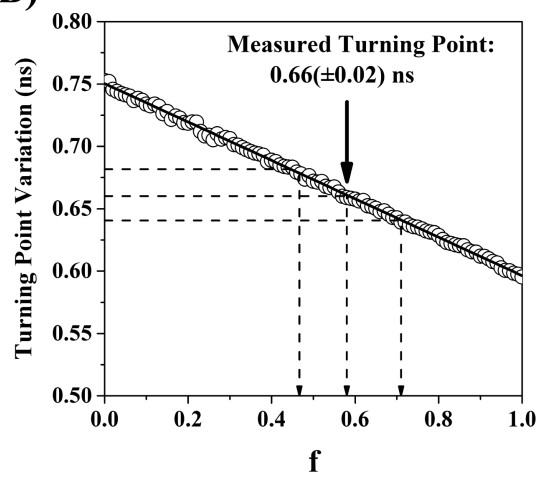

Figure 5. (A) The turning point of the theoretical acceptor window fluorescence decay based on eq 3 is sensitive to changes in the proportion of FRET to the two states available in GST-mC. (B) In numerical simulations, the turning point position decreased linearly as the fraction of interacting acceptors in the short lifetime state, $f$, was varied from 0 to 1 . The experimentally observed turning point of $0.66( \pm 0.02) \mathrm{ns}$ therefore implied that $60( \pm 10) \%$ of the acceptors were in the short lifetime state, reflecting that observed with optical excitation.

dependence of the turnover point on the fraction of FRET to each state. This relationship was theoretically verified by using MATLAB (The Mathworks, USA) to numerically solve eq 3 for its turning point as the proportion of FRET to states 1 and 2 was varied. The turning point was linearly correlated with the fraction of total donors interacting with acceptor state $1, f$, with a gradient of $-0.1373( \pm 0.005) \mathrm{ns}$ and a $y$-intercept (all FRET to state $2, f=0)$ of $0.7157( \pm 0.0003)$ ns. However, this solution neglects the impact of any potential artifacts introduced by the presence of an IRF in real acceptor window fluorescence decay data. We therefore performed numerical simulations of the iterative reconvolution fitting process on simulated data sets (Supporting Information Appendix S11). It was confirmed that the turning point of the data decreased linearly with $f$, as shown in Figure 5B. We observed that the influence of the IRF (fwhm $\sim 60 \mathrm{ps}$ ) was to shift the turning point to later times by, on average, $0.026( \pm 0.005)$ ns. Nonetheless, a linear relationship was maintained, with gradient of $-0.1540( \pm 0.0005) \mathrm{ns}$ and intercept of $0.7504( \pm 0.0003)$ ns. Therefore, based on these simulations, the turning point of $0.66( \pm 0.02)$ ns extracted from the real acceptor window data would imply that $60( \pm 10) \%$ of the interacting acceptors are in the short lifetime state. This mirrors the $57.2( \pm 0.2) \%$ of short lifetime GST-mC present with optical excitation weighted by the donor-acceptor spectral overlap, suggesting that acceptor state selection is completely relaxed in the OG-GSH-GST-mC system.

The anisotropy decay of the FRET mixture in the acceptor window will be an associated combination of the anisotropy 
decays of the sensitized acceptor and the donor bleed through. As shown in Figure 6A, a model in which the donor-acceptor
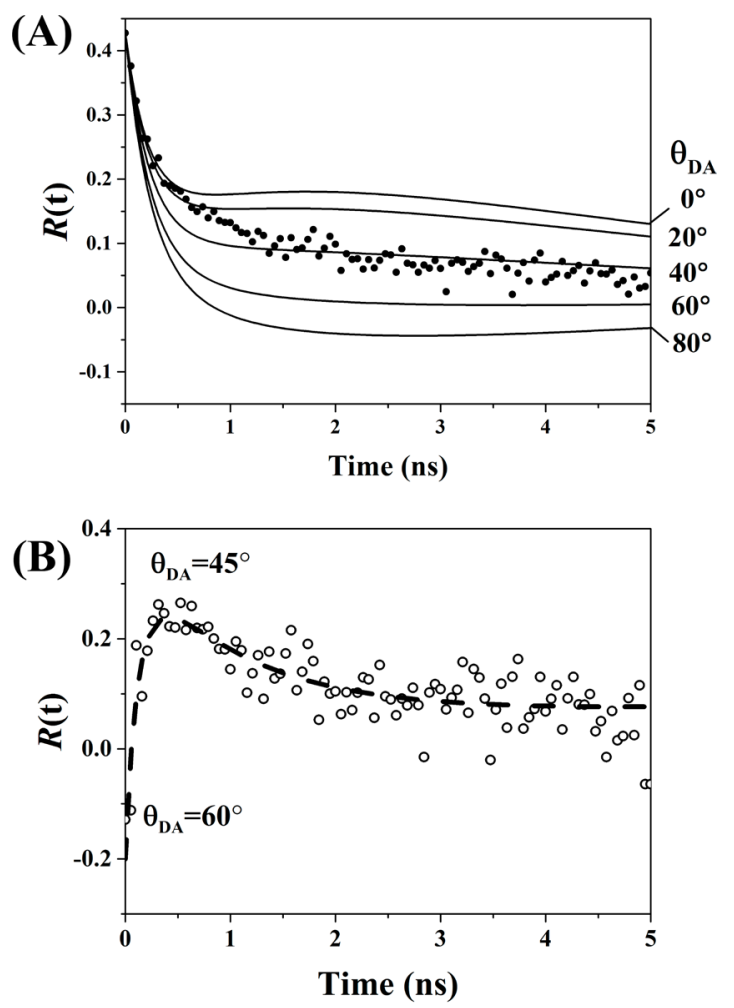

(C)

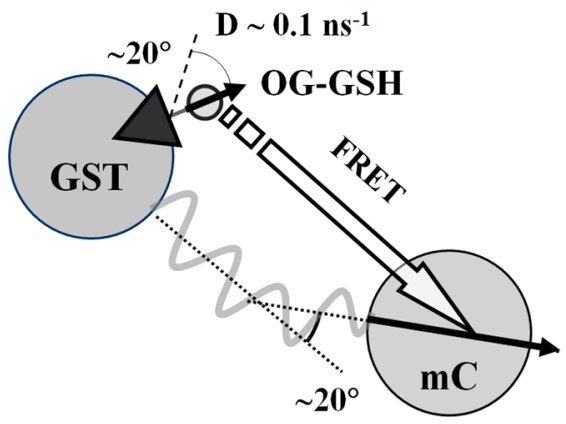

Figure 6. (A) Fluorescence anisotropy decay of a mixture of $60 \mu \mathrm{M}$ OG-GSH and $30 \mu \mathrm{M}$ GST-mC with $880 \mathrm{~nm}$ excitation and detection in the acceptor window $(630-650 \mathrm{~nm})$. The fixed dipole angle model cannot be fit to the data with $\chi_{\mathrm{R}}{ }^{2}$ minimized at an unacceptable value of 3.05 with $\theta_{\mathrm{DA}} \sim 40^{\circ}$. (B) Predicted form of the intrinsic acceptor anisotropy by subtraction of the calculated donor bleed through from the composite anisotropy measured in (A). The initial rise, plateau, and decay imply a time-varying $\theta_{\mathrm{DA}}$ consistent with the measured orientational freedom of both the donor and acceptor. (C) Schematic structure of the OG-GSH-GST-mC complex, the negative initial anisotropy recovered in (B) implies a close to $60^{\circ}$ value for $\theta_{\mathrm{DA}}$.

angle $\theta_{\mathrm{DA}}$ remains static over the time scales at which FRET occurs (Supporting Information Appendix S12) could not be fit to the measured data set. For example, the model giving the lowest $\chi_{\mathrm{R}}^{2}\left(\theta_{\mathrm{DA}}=40^{\circ}\right)$ fitted well to the tail of the decay, but the anisotropy was greatly underestimated at delay times below 1.5 ns. Thus, it is likely that the functional form of the sensitized acceptor fluorescence anisotropy decay was made more complex due to the orientational freedom of the donor.

The form of $R_{\mathrm{A}}{ }^{\mathrm{I}}(t)$ was estimated by performing an intensityweighted subtraction of the theoretical donor window anisotropy contribution predicted from the experimental data (Figure 6B) and also from the best (arbitrary) mathematical representation of the data provided by a three exponential fit $\left(\chi_{\mathrm{R}}{ }^{2}=1.1\right)$, yielding the dashed line in Figure $6 \mathrm{~B}$, as detailed in Supporting Information Appendix S12. $R_{\mathrm{A}}{ }^{\mathrm{I}}(t)$ is characterized by a negative anisotropy $(-0.13)$ at early time followed by a rise peaking around $0.4 \mathrm{~ns}$ later at approximately 0.24 , followed by a slower decay. Based on the approach of Lipari and Szabo, ${ }^{40}$ the diffusion coefficient of the donor motion in OG-GSH-GST$\mathrm{mC}$ was calculated to be the same order of magnitude as $k_{\mathrm{FRET}}$ $\left(0.1 \mathrm{~ns}^{-1}\right.$ compared to $\left.0.5 \mathrm{~ns}^{-1}\right)$. In this regime, an analytical model for $R_{\mathrm{A}}^{\mathrm{I}}(t)$ developed by Tanaka et al. ${ }^{35}$ is seen to be sensitive to the relative orientations of the donor and acceptor rotation axes and their diffusion coefficients ${ }^{41,42}$ yielding rise and decay characteristics similar to Figure 6B. At early times $R_{\mathrm{A}}{ }^{\mathrm{I}}(t)$ will largely correspond to the orientational dynamics of FRET transfer (i.e., a time evolving $\theta_{\mathrm{DA}}$ ), and at later times the intrinsic rotational dynamics of $\mathrm{mC}$ will play a larger role. The early time value of $R_{\mathrm{A}}^{\mathrm{I}}(t)$ (via Supporting Information Equation S49) thus corresponds to an initial value for $\theta_{\mathrm{DA}}$ of around $60^{\circ}$ decreasing to $45^{\circ}$ as shown in Figure 6B. These observations, while largely qualitative, indicate that the FRET interaction is far from static, reflecting the orientational freedom of both donor and acceptor in the GSH-GST complex (Figure 6C). Approaches such as molecular dynamics simulations of $R_{\mathrm{A}}{ }^{\mathrm{I}}(t)$ as recently demonstrated by Nunthaboot et al. ${ }^{43}$ may allow a more quantitative investigation of this system.

\section{CONCLUSIONS}

By combining time-resolved fluorescence intensity and anisotropy measurements of the OG-GSH-GST-mC complex, we have shown that the state restriction observed in fluorescent protein to fluorescent protein FRET is relaxed when the donor is replaced by a more mobile synthetic fluorophore. This demonstrates that the restricted geometry of a fluorescent protein tandem construct, which will remain effectively static on the time scales over which FRET occurs, is a significant cause of the differential energy transfer dynamics between the heterogeneous excited state populations. ${ }^{15}$ As such, the precise configuration of a fluorescent protein FRET pair will not only govern the fluorescence decay rate of the interacting donor but also cause the fluorescence lifetime and quantum yield of the sensitized acceptor to differ from that observed with direct optical excitation. Failure to account for these phenomena in both intensity-based and time-resolved fluorescence studies could therefore result in greatly inaccurate quantitative measurements of interacting fractions and FRET efficiencies.

In the OG-GSH-GST-mC system, the rapid depolarization of the donor fluorescence ruled out use of the (composite) acceptor window anisotropy as a means of probing the acceptor population dynamics. A new approach to the analysis of the acceptor window fluorescence intensity showed that the measured turnover point of the data was sensitive to the proportion of FRET to each state to within $\pm 10 \%$, a degree of precision largely determined by the $\sim 0.6 \mathrm{~ns}$ separation of the two acceptor lifetimes. As the turning point is sensitive to each parameter describing the FRET interaction (Supporting Information Equation S45), and only relies on a simple biexponential fit, this method of analysis could be applicable in situations where FRET dynamics require extraction from lower signal-to-noise data, as for example in live cell fluorescence lifetime imaging microscopy (FLIM). 
Despite the apparent relaxation of state restriction in FRET to $\mathrm{mC}$, donor window anisotropy measurements revealed the presence of a bound but FRET-inactive OG-GSH population pointing to the presence of FRET inactive $\mathrm{MC}$. FRET inactive acceptor states have previously been ascribed to "nonmatured" protein populations with altered spectral characteristics, protonated forms of the fluorophore, photoconversion during the FRET process, or excited-state blinking. ${ }^{10-12}$ Vogel et al. considered the existence of unfavorable, static donor-acceptor geometries as the most likely cause of incomplete FRET between fluorescent proteins. ${ }^{12}$ Our work points to this as the major cause of FRET restriction between EGFP and $\mathrm{mC}$ in the PDK1 homodimer. ${ }^{15}$ However, the discovery of a noninteracting population of OG-GSH-GST-mC complexes points to a continuing role for both photophysical and protein structural heterogeneity as factors for consideration in the interpretation of incomplete FRET with fluorescent proteins.

In conclusion, we have shown that energy transfer restrictions are relaxed by increased angular freedom in the molecular frame, emphasizing the importance of the local donor-acceptor geometry in controlling fluorescent protein FRET. Nevertheless, incomplete energy transfer in OG-GSHGST-mC remained, the detection of which was made possible through donor window anisotropy measurements, a nonstandard FRET technique. Our work demonstrates both the advantage and necessity of a full understanding of the photophysics and the local environment of each donoracceptor pair and also the critical importance of performing spectrally resolved intensity and anisotropy decay measurements in the accurate quantification of fluorescent protein FRET.

\section{ASSOCIATED CONTENT}

\section{S Supporting Information}

The Supporting Information is available free of charge on the ACS Publications website at DOI: 10.1021/acs.jpcc.6b11235.

Extended methods and discussion, absorption and emission spectra, fluorescence and anisotropy decay fits and data tables, turning point modeling and supporting references (Appendices S1-S12, Figures S1-S19, and Tables S1-S4) (PDF)

\section{AUTHOR INFORMATION}

\section{Corresponding Author}

*E-mail: a.bain@ucl.ac.uk.

\section{ORCID ${ }^{\circ}$}

WeiYue Chen: 0000-0001-5004-4376

Angus J. Bain: 0000-0001-8705-8536

\section{Present Address}

${ }^{\#}$ Cancer Cell Biology \& Imaging, King's College London, New Hunt's House, Newcomen Street, London SE1 1UL, United Kingdom.

\section{Notes}

The authors declare no competing financial interest.

\section{ACKNOWLEDGMENTS}

Work at UCL was supported through BBSRC grant BB/ L020874/1. W.Y.C. was funded by the China Scholarship Council (CSC) and Cambridge Trust (CCEIT). C.F.K. acknowledges Wellcome Trust, MRC, and EPSRC funding. This project was initiated during the inaugural Professor Anne
Warner postdoctoral fellowship awarded to T.S.B. through the CoMPLEX Doctoral Training Centre at UCL. We dedicate this paper to the memory of Anne Warner in recognition of her unique and pioneering role in supporting interdisciplinary research and developing postgraduate training at the physical/ life sciences interface.

\section{REFERENCES}

(1) Scholes, G. D. Long-Range Resonance Energy Transfer in Molecular Systems. Annu. Rev. Phys. Chem. 2003, 54, 57-87.

(2) Akrap, N.; Seidel, T.; Barisas, B. G. Förster Distances for Fluorescence Resonant Energy Transfer between mCherry and Other Visible Fluorescent Proteins. Anal. Biochem. 2010, 402 (1), 105-106.

(3) Vafabakhsh, R.; Levitz, J.; Isacoff, E. Y. Conformational Dynamics of a Class C G-Protein-Coupled Receptor. Nature 2015, 524 (7566), 497-501.

(4) Yang, P. S.; Johny, M. B.; Yue, D. T. Allostery in $\mathrm{Ca}^{2+}$ Channel Modulation by Calcium-Binding Proteins. Nat. Chem. Biol. 2014, 10 (3), 231-238.

(5) Patterson, G. H.; Piston, D. W.; Barisas, B. G. Förster Distances between Green Fluorescent Protein Pairs. Anal. Biochem. 2000, 284 (2), 438-440.

(6) Piston, D. W.; Kremers, G.-J. Fluorescent Protein FRET: The Good, the Bad and the Ugly. Trends Biochem. Sci. 2007, 32 (9), 407414.

(7) Rizzo, M. A.; Springer, G. H.; Granada, B.; Piston, D. W. An Improved Cyan Fluorescent Protein Variant Useful for FRET. Nat. Biotechnol. 2004, 22 (4), 445-449.

(8) Borst, J. W.; Hink, M. A.; van Hoek, A.; Visser, A. J. W. G. Effects of Refractive Index and Viscosity on Fluorescence and Anisotropy Decays of Enhanced Cyan and Yellow Fluorescent Proteins. J. Fluoresc. 2005, 15 (2), 153-160.

(9) Hess, S. T.; Sheets, E. D.; Wagenknecht-Wiesner, A.; Heikal, A. A. Quantitative Analysis of the Fluorescence Properties of Intrinsically Fluorescent Proteins in Living Cells. Biophys. J. 2003, 85 (4), 25662580.

(10) Visser, A. J. W. G.; Laptenok, S. P.; Visser, N. V.; van Hoek, A.; Birch, D. J. S.; Brochon, J.-C.; Borst, J. W. Time-Resolved FRET Fluorescence Spectroscopy of Visible Fluorescent Protein Pairs. Eur. Biophys. J. 2010, 39 (2), 241-253.

(11) Millington, M.; Grindlay, G. J.; Altenbach, K.; Neely, R. K.; Kolch, W.; Bencina, M.; Read, N. D.; Jones, A. C.; Dryden, D. T. F.; Magennis, S. W. High-Precision FLIM-FRET in Fixed and Living Cells Reveals Heterogeneity in a Simple CFP-YFP Fusion Protein. Biophys. Chem. 2007, 127 (3), 155-164.

(12) Vogel, S. S.; Nguyen, T. A.; van der Meer, B. W.; Blank, P. S. The Impact of Heterogeneity and Dark Acceptor States on FRET: Implications for Using Fluorescent Protein Donors and Acceptors. PLoS One 2012, 7 (11), e49593.

(13) Laptenok, S. P.; Borst, J. W.; Mullen, K. M.; van Stokkum, I. H. M.; Visser, A. J. W. G.; van Amerongen, H. Global Analysis of Förster Resonance Energy Transfer in Live Cells Measured by Fluorescence Lifetime Imaging Microscopy Exploiting the Rise Time of Acceptor Fluorescence. Phys. Chem. Chem. Phys. 2010, 12 (27), 7593-7602.

(14) Masters, T. A.; Calleja, V.; Armoogum, D. A.; Marsh, R. J.; Applebee, C. J.; Laguerre, M.; Bain, A. J.; Larijani, B. Regulation of 3Phosphoinositide-Dependent Protein Kinase 1 Activity by Homodimerization in Live Cells. Sci. Signaling 2010, 3 (145), ra78.

(15) Masters, T. A.; Marsh, R. J.; Armoogum, D. A.; Nicolaou, N.; Larijani, B. B.; Bain, A. J. Restricted State Selection in Fluorescent Protein Forster Resonance Energy Transfer. J. Am. Chem. Soc. 2013, 135 (21), 7883-7890.

(16) Erickson, M. G.; Alseikhan, B. A.; Peterson, B. Z.; Yue, D. T. Preassociation of Calmodulin with Voltage-Gated Ca2+ Channels Revealed by FRET in Single Living Cells. Neuron 2001, 31 (6), 973985 . 
(17) Gordon, G. W.; Berry, G.; Liang, X. H.; Levine, B.; Herman, B. Quantitative Fluorescence Resonance Energy Transfer Measurements Using Fluorescence Microscopy. Biophys. J. 1998, 74 (5), 2702-2713.

(18) Chen, H.; Puhl, H. L.; Koushik, S. V.; Vogel, S. S.; Ikeda, S. R. Measurement of FRET Efficiency and Ratio of Donor to Acceptor Concentration in Living Cells. Biophys. J. 2006, 91 (5), L39-L41.

(19) Hoppe, A.; Christensen, K.; Swanson, J. A. Fluorescence Resonance Energy Transfer-Based Stoichiometry in Living Cells. Biophys. J. 2002, 83 (6), 3652-3664.

(20) Nagy, P.; Bene, L.; Hyun, W. C.; Vereb, G.; Braun, M.; Antz, C.; Paysan, J.; Damjanovich, S.; Park, J. W.; Szöllsi, J. Novel Calibration Method for Flow Cytometric Fluorescence Resonance Energy Transfer Measurements between Visible Fluorescent Proteins. Cytometry, Part A 2005, 67A (2), 86-96.

(21) Elder, A.; Domin, A.; Kaminski Schierle, G.; Lindon, C.; Pines, J.; Esposito, A.; Kaminski, C. A Quantitative Protocol for Dynamic Measurements of Protein Interactions by Forster Resonance Energy Transfer-Sensitized Fluorescence Emission. J. R. Soc., Interface 2009, 6 (Suppl_1), S59-S81.

(22) Masters, T. A. Time-Resolved Fluorescence Studies of Enhanced Green Fluorescent Protein and the Molecular Dynamics of 3Phosphoinositide Dependent Protein Kinase 1, Dr. Thesis, UCL (University Coll. London), 2009.

(23) Dale, R. E.; Eisinger, J.; Blumberg, W. E. The Orientational Freedom of Molecular Probes. The Orientation Factor in Intramolecular Energy Transfer. Biophys. J. 1979, 26 (2), 161-193.

(24) Chen, W.; Avezov, E.; Schlachter, S. C.; Gielen, F.; Laine, R. F.; Harding, H. P.; Hollfelder, F.; Ron, D.; Kaminski, C. F. A Method to Quantify FRET Stoichiometry with Phasor Plot Analysis and Acceptor Lifetime Ingrowth. Biophys. J. 2015, 108 (5), 999-1002.

(25) Rusinova, E.; Tretyachenko-Ladokhina, V.; Vele, O. E.; Senear, D. F.; Alexander Ross, J. B. Alexa and Oregon Green Dyes as Fluorescence Anisotropy Probes for Measuring Protein-protein and Protein-nucleic Acid Interactions. Anal. Biochem. 2002, 308 (1), 1825 .

(26) Bhunia, D.; Chowdhury, R.; Bhattacharyya, K.; Ghosh, S. Fluorescence Fluctuation of an Antigen-Antibody Complex: Circular Dichroism, FCS and smFRET of Enhanced GFP and Its Antibody. Phys. Chem. Chem. Phys. 2015, 17 (38), 25250-25259.

(27) Müller, C. B.; Loman, A.; Pacheco, V.; Koberling, F.; Willbold, D.; Richtering, W.; Enderlein, J. Precise Measurement of Diffusion by Multi-Color Dual-Focus Fluorescence Correlation Spectroscopy. EPL (Europhysics Lett. 2008, 83 (4), 46001.

(28) Armstrong, R. N. Glutathione S-Transferases: Reaction Mechanism, Structure, and Function. Chem. Res. Toxicol. 1991, 4 (2), 131-140.

(29) Smith, D. B.; Johnson, K. S. Single-Step Purification of Polypeptides Expressed in Escherichia Coli as Fusions with Glutathione S-Transferase. Gene 1988, 67 (1), 31-40.

(30) Harper, S.; Speicher, D. W. Purification of Proteins Fused to Glutathione S-Transferase. Methods Mol. Biol. 2011, 681, 259-280.

(31) Becker, W. Advanced Time-Correlated Single Photon Counting Applications; 2015.

(32) Blacker, T. S.; Marsh, R. J.; Duchen, M. R.; Bain, A. J. Activated Barrier Crossing Dynamics in the Non-Radiative Decay of NADH and NADPH. Chem. Phys. 2013, 422, 184-194.

(33) Köllner, M.; Wolfrum, J. How Many Photons Are Necessary for Fluorescence-Lifetime Measurements? Chem. Phys. Lett. 1992, 200 (1-2), 199-204.

(34) White, S. S.; Li, H.; Marsh, R. J.; Piper, J. D.; Leonczek, N. D.; Nicolaou, N.; Bain, A. J.; Ying, L.; Klenerman, D. Characterization of a Single Molecule DNA Switch in Free Solution. J. Am. Chem. Soc. 2006, 128 (35), 11423-11432.

(35) Tanaka, F.; Mataga, N. Dynamic Depolarization of Interacting Fluorophores. Effect of Internal Rotation and Energy Transfer. Biophys. J. 1982, 39 (2), 129-140.

(36) Ludescher, R. D.; Peting, L.; Hudson, S.; Hudson, B. TimeResolved Fluorescence Anisotropy for Systems with Lifetime and Dynamic Heterogeneity. Biophys. Chem. 1987, 28 (1), 59-75.
(37) Kremers, G.-J.; Hazelwood, K. L.; Murphy, C. S.; Davidson, M. W.; Piston, D. W. Photoconversion in Orange and Red Fluorescent Proteins. Nat. Methods 2009, 6 (5), 355-358.

(38) Macdonald, P. J.; Chen, Y.; Mueller, J. D. Chromophore Maturation and Fluorescence Fluctuation Spectroscopy of Fluorescent Proteins in a Cell-Free Expression System. Anal. Biochem. 2012, 421 (1), 291-298.

(39) Blacker, T. S.; Duchen, M. R.; Bain, A. J. Heterogeneity and Restricted State Selection in FRET with Fluorescent Proteins; International Society for Optics and Photonics: 2016; p 971401.

(40) Lipari, G.; Szabo, A. Effect of Librational Motion on Fluorescence Depolarization and Nuclear Magnetic Resonance Relaxation in Macromolecules and Membranes. Biophys. J. 1980, 30 (3), 489-506.

(41) Tanaka, F. Theory of Time-Resolved Fluorescence under the Interaction of Energy Transfer in a Bichromophoric System: Effect of Internal Rotations of Energy Donor and Acceptor. J. Chem. Phys. 1998, 109 (3), 1084.

(42) Tanaka, F. Effects of Internal Rotations on the Time-Resolved Fluorescence in a Bichromophoric Protein System Under the Energy Transfer Interaction. J. Fluoresc. 2000, 10 (1), 13-20.

(43) Nunthaboot, N.; Tanaka, F.; Kokpol, S.; Visser, N. V.; van Amerongen, H.; Visser, A. J. W. G. Molecular Dynamics Simulation of Energy Migration between Tryptophan Residues in Apoflavodoxin. RSC Adv. 2014, 4 (59), 31443. 